\title{
El Caso de Francisca y el Sentido Otorgado a los Números Decimales
}

\section{The Case of Francisca and the Sense Attributed to Decimals Numbers}

\author{
Marta Elena Valdemoros-Álvarez ${ }^{*}$ \\ Elena Fabiola Ruiz-Ledesma**
}

\begin{abstract}
Resumen
En una investigación cualitativa realizada en una escuela primaria nocturna de la Ciudad de México, desarrollamos este estudio de caso referido a una joven de 19 años, incorporada al quinto grado de esa institución y dependiente de una papelería. Ella resolvió problemas que involucraban el uso de los números decimales y sus operaciones fundamentales. Francisca fue seleccionada a partir de la aplicación de un cuestionario en el que la joven evidenció un desempeño globalmente adecuado, aunque no exento de errores. En el seguimiento del caso de Francisca fueron utilizados dos instrumentos metodológicos: el cuestionario y la entrevista; la última y algunos de sus diálogos fueron el soporte fundamental de este estudio. Nuestra pregunta general de investigación fue: ¿cómo asigna sentido a los números decimales el adulto, cuando resuelve problemas referidos a tales números? Francisca los significó a partir de las situaciones familiares asociadas a sus experiencias de vida y de trabajo; en la resolución de problemas, les asignó sentido al establecer equivalencias entre tales números y los naturales, en primera instancia, aunque también los vinculó con las fracciones. Al idear problemas, la joven privilegió el uso del dinero y de ciertas medidas de longitud expresadas en el sistema métrico decimal. Algunas dificultades de Francisca se mostraron en las interpretaciones posicionales de las cifras decimales y de la notación del punto decimal. Ella exhibió unos pocos errores de lectura, escritura y operacionales ligados a ciertas nociones posicionales inestables.
\end{abstract}

Palabras-clave: Decimales. Sentido. Significación. Punto Decimal ${ }^{* * *}$ (Coma Decimal). Resolución de Problemas.

\begin{abstract}
In a qualitative research carried out in a night elementary school in Mexico City, we performed the case study of a young woman of 19 years of age and fifth grade student at the school, who is a stationery's employee. She solved problems, which involved the use of decimal numbers and their basic operations. Francisca chose from a questionnaire in which she evidenced an adequate overall performance although not without errors. Two followup instruments were used in Francisca's case: questionnaire and interview; the latter and some of its dialogs were the fundamental support of this research. Our general research question was: how does the adult assign sense to decimal numbers in the resolution of problems with those numbers? Francisca signified such numbers through familiar situations of life and working experiences. In problem solving, she assigned decimal numbers establishing equivalence between those numbers and mainly natural numbers, while she linked secondly decimal

\footnotetext{
"Doctorado otorgado por el Centro de Investigación y de Estudios Avanzados del IPN (Cinvestav del IPN). Investigadora titular $3 \mathrm{C}$ y profesora de Maestría y Doctorado el Centro de Investigación y de Estudios Avanzados del IPN (Cinvestav del IPN), México, México. Dirección Postal: Av. Politécnico Nacional 2508, Col. San Pedro Zacatenco, Gustavo A. Madero, C. P. 07360, México, México. E-mail: mvaldemo@cinvestav.mx.

*** Doctorado otorgado por el Centro de Investigación y de Estudios Avanzados del IPN (Cinvestav del IPN). Docente de Matemáticas titular "C", e Investigadora en el área de Cómputo Educativo en el Instituto Politécnico Nacional. Escuela Superior de Cómputo (ESCOM-IPN), México, México. Dirección Postal: Av. Othón de Mendizábal s/n, Col. La Escalera, Gustavo A. Madero, C.P 07320, México, México. E-mail: efruiz@ipn.mx; elen_fruiz@yahoo.com.mx.

*** En México las cifras decimales son antecedidas por un punto decimal (o sea, no por una coma decimal).
} 
numbers with fractions. When this young woman produced new problems, she favored the use of money and length measures represented by the decimal metric system. Some of Francisca's difficulties were referred to the interpretations of the decimal positions and decimal notations. Also, she made mistakes in reading, writing, or in operations linked to certain unstable positional notions.

Keywords: Decimals. Sense. Signification. Decimal Point (Decimal Comma). Problem Solving.

\section{Problema y preguntas de investigación}

\subsection{El problema de investigación}

El objeto de estudio que aquí identificamos es la elaboración de sentido por parte del adulto en proceso de formación básica, en el ámbito de la resolución de problemas elementales, utilizando los números decimales y sus operaciones fundamentales. Dicha elaboración de sentido sienta las bases de la comprensión (la cual suele permanecer limitada en muchos sujetos que tienden a apoyarse sólo en la poderosa algoritmia de los decimales) y el punto de partida de la ulterior construcción de conceptos referidos a tales números (de estos últimos objetos de estudio no nos ocupamos en la presente investigación).

En el proceso semántico de producción de sentido concedemos especial atención a las dificultades cognitivas vinculadas a la resolución de tales problemas (en tanto indicadoras de algunos nodos semánticos primordiales) y a los errores manifiestos en la mencionada ejecución (por su carácter de producciones reveladoras de la interpretación otorgada a los números decimales por quien da solución a la tarea, de acuerdo con CENTENO, 1988).

Involucramos a la resolución de problemas en nuestra indagación porque es el terreno en el que el sujeto, de un modo no deliberado, pone a prueba sus ideas de manera intuitiva y empírica, confrontándolas unas con otras, depurándolas y generando nuevos sentidos para sus acciones. Esto lo planteamos, apegándonos a los procesos atribuidos a la resolución de problemas matemáticos por Nesher (1982, 1992), Vergnaud (1982), Carpenter, Fennema y Romberg (1993), Kieren (1993), Valdemoros y Ruiz (2008), Deliyanni et al. (2009), entre muchos investigadores de la aritmética que han desarrollado este recurso.

Al situar a los números decimales en el terreno de la escuela primaria de adultos, estamos enfatizando el carácter elemental de su desarrollo cognitivo inicial y la correspondiente relevancia de su investigación.

\subsection{Las preguntas de investigación}


En íntima relación con el problema de investigación planteado, y tomando en consideración las aportaciones de Bruner y Haste (1990), referidas a que la elaboración de sentido es esencialmente una decodificación por la cual se abre camino el pensamiento, el cual se expresa mediante un rico repertorio de procesos semánticos, formulamos, a continuación, nuestra pregunta de investigación fundamental.

a) ¿Cómo asigna sentido a los decimales el adulto que resuelve problemas con dichos números?

En cuanto a la producción de sentido, interesa interpretar qué piensa el sujeto cuando avanza hacia la solución de un problema aritmético elemental con números decimales, qué procesos de significación parece privilegiar, qué referentes concretos respaldan sus elaboraciones. Todo ello da cuenta de la comprensión a la que accede el sujeto, en torno a dichos números.

Apoyándonos en el planteamiento ya identificado de Bruner y Haste (1990), prevemos que la asignación de sentido a los números decimales, por parte del adulto, sea identificable a través de los procesos de pensamiento (es decir, de las reflexiones personales) que el sujeto comunique, de los procesos de significación fundamentales que desarrolle (o sea, del otorgamiento de significado al significante decimal dado, según DUCROT; TODOROV, 1981) y de los referentes concretos (los objetos y situaciones reales con los que el individuo relacione al signo, conforme a DUCROT y TODOROV, 1981) en los que base sus elaboraciones semánticas. En los tres últimos procesos y contenidos cognitivos (íntimamente vinculados entre sí) se funda el análisis de resultados, cuando los datos a ellos asociados sean susceptibles de reconocimiento.

b) ¿Qué dificultades cognitivas enfrenta el adulto en la aludida elaboración de sentido?

La nueva pregunta deriva de la anterior y se adecua al propósito de detectar las situaciones de la resolución que sean más inaccesibles para el sujeto, procurando caracterizar la naturaleza de los obstáculos cognitivos involucrados en ellas, hasta donde sea posible.

c) ¿Qué errores manifiesta el adulto en la resolución de problemas, como un modo particular de asignación de sentido?

La indagación que hemos perfilado, también la hacemos extensiva a los errores, los que agregan el punto de vista del sujeto ante los obstáculos que no ha podido superar y respecto a los cuales requiere reflexionar.

\section{Marco teórico}


Los rubros señalados en cada subtítulo de la presente sección guardan íntima relación con el problema y las preguntas de investigación que aquí nos planteamos, razón por la cual decidimos presentar de esta manera nuestros antecedentes teóricos más destacados.

\subsection{Los números decimales, sus operaciones y las dificultades cognitivas a ellos asociadas}

En el desarrollo de una investigación referida a las concepciones que subyacen en la resolución de problemas con números decimales, Bell, Swan y Taylor (1981) afirman que, sobre dicha resolución y sobre la comprensión de quien procura tal solución, pesan el tipo de operación que se realice, el tamaño y complejidad de los números involucrados, la familiaridad de la situación planteada por el problema aritmético y la legibilidad de su texto.

En relación al tipo de operación a realizar, Bell, Swan y Taylor (1981) consideran más accesibles a la adición y sustracción, en tanto que más dificultosas a la multiplicación y la división (siendo, además, más complejas las operaciones inversas que las operaciones directas); a estos planteamientos les agregan las dificultades ligadas a la estructura misma del problema a resolver (es más inaccesible un problema multiplicativo de proporcionalidad múltiple que cualquier otra clase de problemas multiplicativos). A modo de conclusión del estudio aludido, estos investigadores enfatizan que es muy importante el trabajo o las elaboraciones que el sujeto realice respecto a la selección de la operación adecuada, en tanto nos proporciona evidencias de procesos subyacentes.

En cuanto a las operaciones fundamentales, Centeno (1988) afirma que la extensión de éstas desde los naturales hacia los números decimales requiere del planteamiento de nuevas situaciones didácticas que otorguen significado a dichas operaciones. En particular, dicha autora reconoce que el desarrollo de la división permite reorganizar las restantes operaciones y dar sentido pleno al orden de los números decimales. No menos importante para la comprensión de los decimales resulta que el sujeto del aprendizaje conozca muchas formas equivalentes de identificar un número decimal.

En contraste con el planteamiento anterior, Okazaki (2003) afirma que en el aprendizaje de las operaciones con números decimales, los estudiantes tienden a privilegiar los procedimientos algorítmicos asociados a dichas operaciones, permaneciendo relegadas las elaboraciones del pensamiento, las que requieren ser fortalecidas a través de una enseñanza pertinente.

Tomando en cuenta todo lo aportado por los mencionados investigadores, nosotros procuramos apoyarnos en estos aspectos a la hora de diseñar los problemas incorporados al 
cuestionario y a la entrevista, para lograr en ellos una presentación acorde con las posibilidades cognitivas de nuestros sujetos. Por otra parte, atendiendo a las reflexiones contenidas en los dos últimos párrafos, el análisis de los resultados de la presente investigación está orientado hacia la detección de los procesos de pensamiento que se desarrollan en la resolución de problemas aditivos y multiplicativos como para poder dar respuesta a los planteamientos diferenciados de Centeno (1988) y Okazaki (2003).

\subsection{El reconocimiento de experiencias familiares y la construcción del sentido}

Con referencia a la familiaridad de los problemas, Bell, Swan y Taylor (1981) la atribuyen a la proximidad que los mismos guarden con la experiencia habitual de quien da solución a la tarea, Además, distinguen los problemas familiares de los problemas abstractos, resultando, los primeros, en una sencilla resolución, y los últimos son de complejo abordaje.

Por su parte, los investigadores abocados a la problemática educativa básica de los jóvenes y adultos afirman que tanto los conocimientos generales, como los conocimientos matemáticos a ser construidos institucionalmente por tales sujetos en proceso de formación elemental, deben partir del cúmulo previo de experiencias logradas en ámbitos no escolarizados, es decir, en los otros espacios vitales en los que se desenvuelven dichas personas y donde, desde un entorno familiar para el sujeto, se construye un saber estable y con sentido (MESSINA, 1993, GARCÍA CARRASCO, 1997, JÓIA, 1997, entre otros).

En coincidencia con lo destacado en último término, Gimeno y Pérez (1992), han caracterizado a las escenificaciones planteadas en el terreno didáctico como una reconstrucción crítica de la realidad (esta última expresión se ajusta al planteamiento de reconstrucción crítica de concepciones y experiencias de GIMENO y PÉREZ, 1992, a la par de coincidir con la reconstrucción de la realidad expresada por SCHWARTZ y JACOBS, 1984), lograda en un ámbito educativo y reproduciendo características fundamentales de aquélla. Todo ello brinda a los jóvenes y adultos un campo simbólico cargado de sentido para el planteamiento y resolución de problemas aplicando números decimales, en los que se planteen situaciones de su vida personal y comunitaria, razón por la cual incorporamos esta aportación en nuestro enfoque del caso de Francisca.

Broitman, Itzcovich y Quaranta (2003) consideran que las situaciones familiares para el sujeto del aprendizaje, en la escuela elemental, son las que involucran el cálculo con dinero y con medidas porque estos contenidos de los números decimales establecen nexos entre el saber previo de los alumnos y los nuevos significados que deberán construir; en especial, el 
cálculo con dinero posibilita el desarrollo de recursos y notaciones espontáneas de los estudiantes, en el tratamiento inicial de tales números. En nuestra interpretación de este planteamiento, el involucrar lo familiar ofrece un puente importante para la generación de sentido en el aprendizaje y la consiguiente comprensión de los contenidos matemáticos abordados.

Con respecto a la producción de sentido, Hunter y Anthony (2003) enfatizan la relevancia de llevar a cabo una enseñanza eficaz que propicie un ambiente adecuado para el aprendizaje matemático, donde el estudiante razone, fundamente sus elaboraciones, explore ideas alternativas, valide y re-organice su pensamiento. Nosotros consideramos que todo eso es alcanzable en el terreno de la resolución de problemas y en la realización de experiencias especiales como el tipo de entrevista que promovemos a través del presente estudio de caso.

\subsection{Los errores vinculados a los números decimales}

Centeno (1988) plantea que los errores cometidos al hacer uso de los números decimales permiten un interesante acercamiento a cómo el sujeto cognoscente concibe dichos números y qué sentido les otorga, lo cual acentúa la relevancia que tiene una adecuada exploración de dichos errores. En lo fundamental, los errores vinculados a las dificultades de lectura y escritura numérica derivan en distorsiones asociadas al valor posicional de las cifras decimales (esto se evidencia, especialmente, cuando se trata de números menores que la unidad). Los errores asociados a la interpretación del cero se expresan por distintas vías, dependiendo del marco en el que aparezcan (en vinculación con la posición ocupada por el cero con respecto al número en el que se inserta). Son muy variados los errores ligados al orden de los decimales, siendo particularmente notorios aquéllos que suponen un desplazamiento de las ideas de orden desde los enteros hacia los decimales.

Centeno (1988) también destaca la relevancia de los errores asociados a las operaciones fundamentales, en torno a las cuales emergen concepciones tales como que al multiplicar se genera un producto mayor que los factores originales, por citar tan sólo un ejemplo notable.

Steinle y Stacey (2004) señalan que de los muchos errores reconocidos por la investigación previa, con respecto al uso de números decimales, el rasgo común a todos ellos es que tienden a expresarse como sistemáticos y predecibles; en otras palabras, esos rasgos identifican a los errores como lo contrario de manifestaciones casuales del sujeto cognoscente. A lo cual hay que agregar que, en el desarrollo de un estudio longitudinal 
llevado a cabo con cientos de estudiantes, Steinle y Stacey (2003) pudieron determinar que diferentes errores persisten a través del tiempo y de distintos niveles de escolaridad.

Asimismo, Boufi y Shaftouru (2002) conceden especial importancia a las condiciones colectivas e individuales en las que los errores emergen, en relación con la enseñanza impartida. Tal reconocimiento deriva de la circunstancia de que los mencionados investigadores consideran que el aprendizaje matemático es tanto social como individual, y que los aspectos relacionales del mismo son importantes.

Una vez más, los antedichos planteamientos en torno a los errores los tomamos en cuenta cuando diseñamos las tareas que integran el cuestionario y la entrevista. A nivel del análisis de los errores detectables en el desempeño de Francisca, procuramos establecer los nexos posibles entre estos últimos y los planteamientos contenidos en la presente sección.

\section{Diseño metodológico}

\subsection{Los sujetos del estudio}

El cuestionario exploratorio fue resuelto por diecisiete jóvenes y adultos de sexto, quinto y cuarto grados, para facilitarnos la selección de los casos a estudiar; ellos tenían edades comprendidas entre 15 y 70 años y desempañaban actividades laborales diversas (obreros, artesanos, vendedores ambulantes, dependientes de comercio, amas de casa y vigilantes).

De los cinco casos conformados, aquí tan sólo presentamos el de Francisca, con 19 años de edad, en quinto grado de la escuela nocturna y empleada en una papelería. El perfil de ella correspondió al de una persona capaz de efectuar clasificaciones de distinta naturaleza, atender comercialmente diferentes demandas de los clientes, realizar el cálculo inicial del monto de cada una de sus ventas, efectuar pedidos a numerosos proveedores de la tienda, entre otras muchas actividades ocupacionales en las que se desarrollan múltiples procesos aritméticos. Escogimos a Francisca, a partir del cuestionario, porque ella exhibió, allí, resultados globalmente adecuados y presumiblemente cargados de significación, a la par de generar algunos errores que concitaron nuestro interés.

\subsection{El escenario}


La investigación se realizó en una escuela primaria nocturna, en un barrio periférico de la Ciudad de México, situado al interior de una zona industrial habitada por trabajadores procedentes de varias regiones rurales del país y dedicados, con posterioridad, a otras ocupaciones. La escuela está conformada por pequeños grupos de adolescentes y adultos incorporados a los seis grados establecidos en todas las instituciones educativas de este tipo, las cuales pertenecen al sistema público escolarizado de enseñanza básica. Las actividades académicas se desarrollan de lunes a viernes, durante dos horas diarias.

\subsection{Los instrumentos metodológicos involucrados}

\subsubsection{El cuestionario inicial}

El mismo asumió una modalidad exploratoria, realizándose luego el análisis cualitativo de sus resultados. Los ocho problemas del cuestionario presentaron una organización común: estuvieron conformados por un breve texto, asociado a ciertos dibujos que propiciaban una adecuada reconstrucción de distintos aspectos referidos a las experiencias vitales de jóvenes y adultos. Dicha organización de las tareas se dio para otorgar gran familiaridad a cada situación descrita y facilitar la lectura de los adultos (quienes suelen presentar dificultades en la decodificación de textos). Para lograr mayor proximidad a la vida de ellos, procuramos realizar un diseño de problemas aritméticos que se ajustara a diversos perfiles de su quehacer laboral, reconstruyendo distintos eventos de su experiencia personal, familiar y comunitaria.

En tales problemas, los sujetos debían comparar y ordenar decimales, sumar y restar decimales, multiplicar un número decimal por un número natural, dividir un número decimal entre un número natural y decidir acerca de las expresiones decimales adecuadas a determinadas situaciones dadas. Las medidas identificadas en los problemas presentados, correspondieron al uso de longitudes, distancias, capacidades, como también, del dinero.

\subsubsection{La entrevista de corte didáctico}

A partir del desempeño en el cuestionario evidenciado por todos los sujetos con los que se llevó a cabo la investigación, fueron escogidos cinco adultos y jóvenes (pertenecientes a cuarto, quinto y sexto grados), para la realización del estudio de casos. 
A posteriori, se llevó a término una entrevista con los cinco alumnos seleccionados, la cual fue el instrumento fundamental del estudio realizado, de corte individual y semiestructurado. En ella, cada estudiante fue sometido a tareas de diseño común, análogas a las incluidas en el cuestionario, de manera que al concluirlas pudiesen ser comparados los resultados de los cinco casos, al final de la investigación. A causa del tiempo disponible para dialogar con cada sujeto, tuvimos que dividir la entrevista en dos partes y llevarlas a cabo en distintas fechas.

Debido al carácter didáctico de la entrevista (de acuerdo con VALDEMOROS, 1998), hubo dos momentos diferenciados en su realización: 1) una fase inicial, orientada por propósitos de exploración de los aspectos y procesos ligados a la resolución de problemas, en la que se procuró determinar el avance de cada sujeto a partir de sus propias iniciativas y 2) una fase ulterior, de carácter constructivista-didáctico, donde el entrevistador propició en el entrevistado la superación de sus dificultades cognitivas, retroalimentándolo, pero evitando sugerir estrategias o interferir en la búsqueda de nuevas soluciones por parte del adulto. En la consecución de la entrevista, fue crucial el pasaje del primero al segundo momento de la misma, motivo por el cual dicho tránsito fue valorado en cada situación y fue llevado a cabo una vez que se agotaron las posibilidades de un comienzo, al efectuarse la exploración inicial.

\subsection{Los procedimientos de validación cualitativa adoptados}

Para legitimar el cuestionario se ejercieron controles cruzados entre dos observadores. Esencialmente, los controles cruzados consisten en el contraste entre dos fuentes distintas de observación para llegar a constatar la persistencia y equilibrio de lo observado, con lo cual se verifica su consistencia (de acuerdo con TAYLOR; BOGDAN, 1987).

Para validar las entrevistas, nosotros decidimos triangular distintos procesos de solución desplegados por Francisca ante problemas aritméticos con números decimales que presentasen una organización similar entre sí. Para ello, apelamos a una triangulación mixta consistente en la comparación entre los diversos métodos usados, los cambios registrados en diferentes momentos de la experiencia de campo y distintas tareas aritméticas desarrolladas (TAYLOR; BOGDAN, 1987; WOODS, 1987; COHEN; MANION, 1990).

\section{Análisis de los resultados obtenidos}

\subsection{Resultados de Francisca en el cuestionario inicial exploratorio}


Esta información la presentamos brevemente en la Figura 1, la cual incluye tanto las tareas aritméticas integradas al cuestionario como las respectivas producciones de la estudiante que constituye nuestro caso, respuestas finales que hemos distinguido al subrayarlas, además de escribirlas con grafías itálicas. Cabe mencionar que en todas las tareas y en su análisis se incluyen las posiciones decimales precedidas por un punto decimal (no por una coma decimal), ya que la primera de estas opciones es la convención adoptada en México y múltiples países (Inglaterra, Estados Unidos, Canadá, Tailandia, Japón, entre otros).

\begin{tabular}{|c|c|}
\hline Tarea & Elaboración de Francisca \\
\hline 1 & 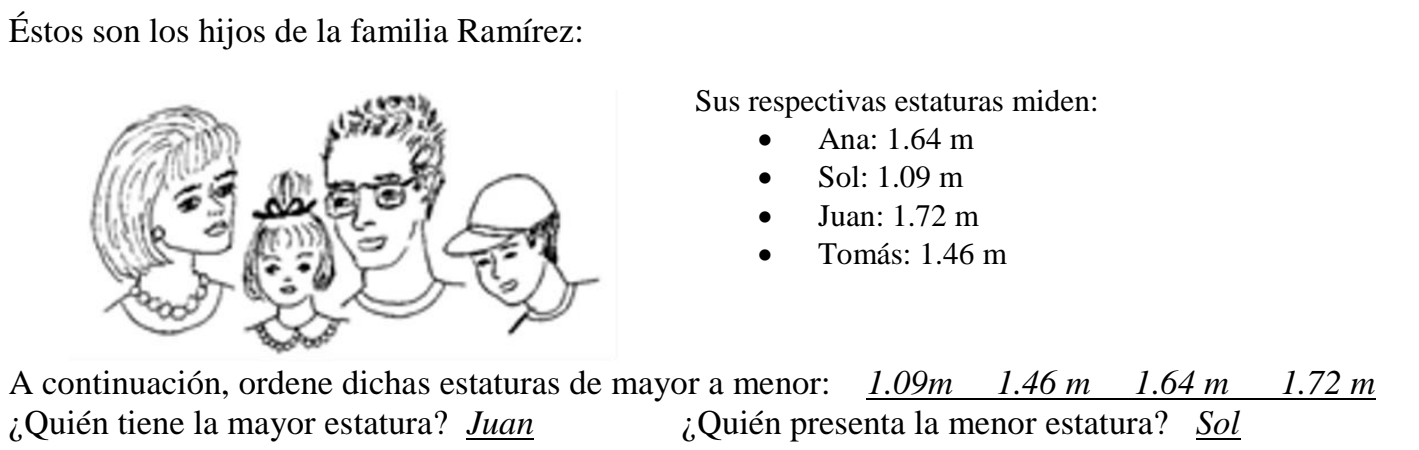 \\
\hline 2 & $\begin{array}{l}\text { Todos los días, un camionero realiza un recorrido de } 8.185 \text { kilómetros, desde su casa hasta } \\
\text { la Central de Abastos. } \\
\text { Con motivo de unos arreglos viales públicos, la próxima semana será desviado de ese camino y } \\
\text { recorrerá } 9.147 \text { kilómetros, desde su casa hasta la Central de Abastos. } \\
\text { El recorrido más largo es de } 9.147 \text { kilómetros. }\end{array}$ \\
\hline 3 & 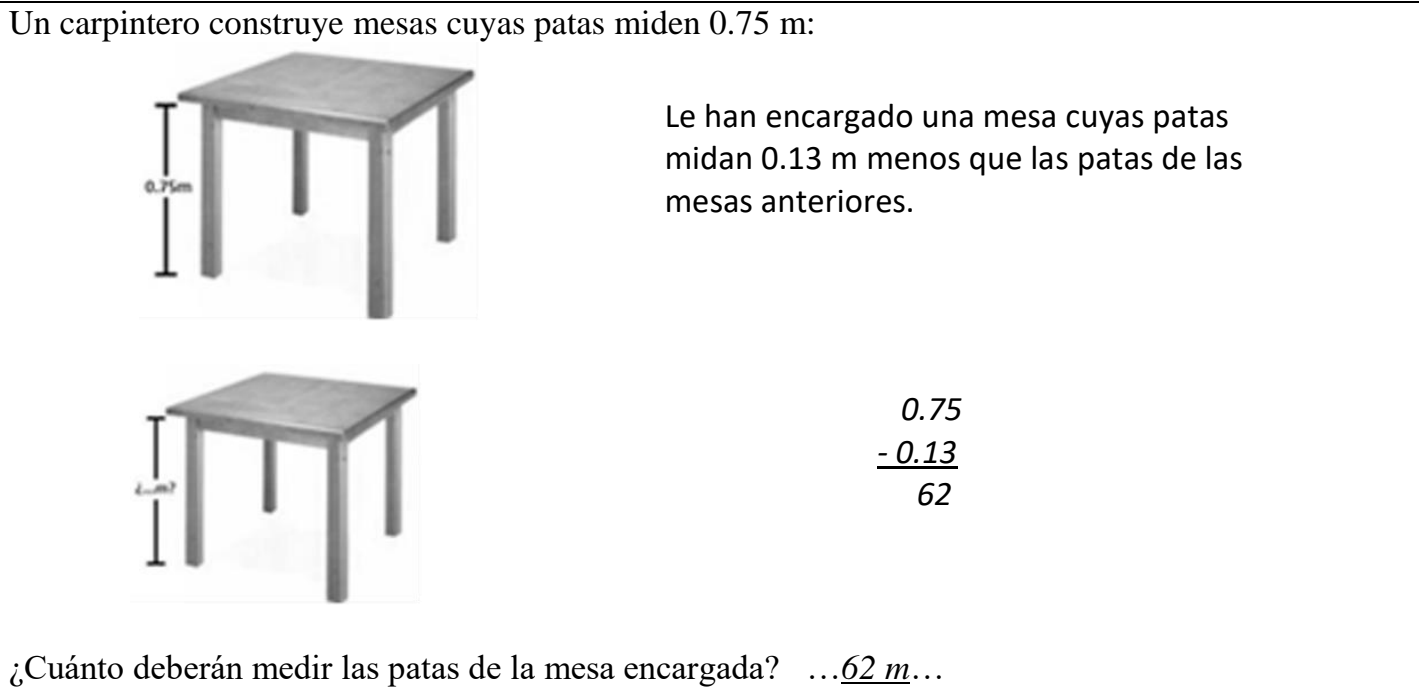 \\
\hline 4 & En una fábrica de camisetas producen una talla grande de esta prenda, cuyo largo es de $0.65 \mathrm{~m}$ : \\
\hline
\end{tabular}




\begin{tabular}{|c|c|}
\hline & $\begin{array}{l}\text { A pedido de sus clientes comenzarán a fabricar una talla extra grande que mida } 0.11 \mathrm{~m} \text { más de largo } \\
\text { que la anterior: } \\
\text { ¿Cuánto medirá el largo de la camiseta extra grande? } \frac{0.11}{0.76} \quad \ldots .\end{array}$ \\
\hline 5 & 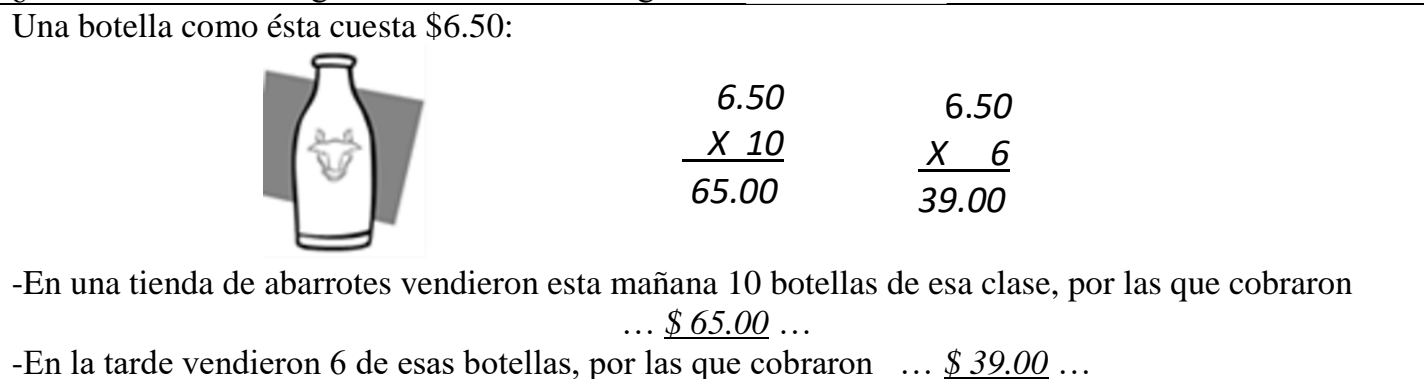 \\
\hline 6 & $\begin{array}{l}\text { Un pintor ha comprado } 1.5 \text { litro de laca para pintar estas sillas: } \\
\text { ¿Cuánta laca destinará a cada silla? ... } \frac{0.3}{\text {. ... litro. }} \\
\text { ¿Cómo llegó usted a ese resultado? } \ldots \text { diviendo } 1.5 \text { entre } 5 \ldots\end{array}$ \\
\hline 7 & $\begin{array}{l}\text { Coloque una cruz junto a la forma como debe ser dicho o leído } 8.185 \text { kilómetros: } \\
\qquad \begin{array}{l}\times \text { Ocho mil ciento ochenta y cinco kilómetros. } \\
\times \text { Ocho, punto, ciento ochenta y cinco kilómetros. } \\
\times \text { Ocho kilómetros, ciento ochenta y cinco metros. }\end{array}\end{array}$ \\
\hline 8 & 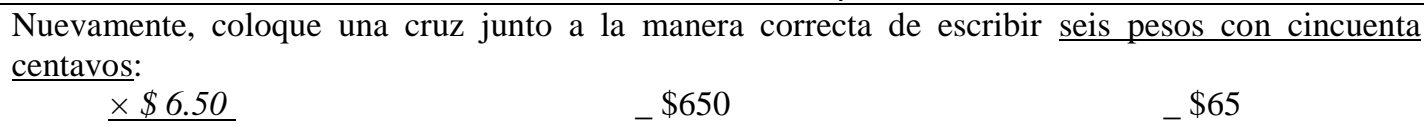 \\
\hline
\end{tabular}

Figura 1- Resultados de Francisca en el cuestionario inicial exploratorio Fuente: contenidos producidos en la investigación de las autoras

Esos ocho problemas tienen el objetivo de: explorar las relaciones de orden entre decimales (Tareas 1 y 2), la sustracción (Tarea 3), la adición (Tarea 4), la multiplicación (Tarea 5), la división (Tarea 6), la lectura adecuada de determinada notación decimal (Tarea 7) y la escritura correcta de cierta expresión decimal (Tarea 8). En seis de estas actividades se usan medidas de distinto tipo; los dos ítems restantes involucran la utilización del dinero. Además, se incluyen algunas dificultades de cálculo: el involucramiento del cero antes del punto decimal, en las medidas dadas por el problema (Tareas 3 y 4) y la obtención de un cociente por parte de la estudiante, el que también se inicia con cero antes del punto decimal (Tarea 6). 


\subsection{Análisis de los resultados del cuestionario inicial exploratorio}

Esta sección está destinada al análisis de la información recogida aquí, ya sea mediante la interpretación de las correspondientes expresiones y algoritmos escritos o escogidos por Francisca (los que en la Figura 1 transcribimos con grafías itálicas y negritas, además de subrayar las mencionadas expresiones) o del planteamiento de nuestros supuestos ligados al cuestionario exploratorio, los que deberían ser confirmadas a posteriori.

En general, el desempeño de Francisca en el cuestionario fue adecuado, aunque incurrió en tres errores cuya naturaleza comentamos seguidamente. Advertirá el lector que la prueba de lápiz y papel nos permitió seguir más de cerca las elaboraciones de la joven en lo referente a los errores, donde dispusimos de más evidencias (en este instrumento).

El primer error fue planteado en la Tarea 1, donde ella procedió adecuadamente en el ordenamiento de las estaturas, pero invirtió el sentido del mismo, el que debía plantearse de mayor a menor y Francisca lo efectuó de menor a mayor; no parece haber aquí más que un descuido de su atención con respecto a las condiciones requeridas en este problema.

El segundo error lo presentó Francisca ante la Tarea 3, en la que desarrolló correctamente el algoritmo de la sustracción pero no respetó el señalamiento del punto decimal en la diferencia resultante, lo que la condujo a reconocer como solución final del problema a $62 \mathrm{~m}$ (o sea, 62 metros), sin que esto llamase su atención ni la indujera a rectificar el señalado resultado. Ante esta dificultad de lectura (la cual se expresa en la interpretación de los datos numéricos dados en el problema, así como de los obtenidos al aplicar el algoritmo), formulamos el supuesto de que, tal vez, las dudas generadas por el despliegue del algoritmo las procuró resolver, evadiendo los números decimales a través de un malogrado intento de establecer una correspondencia con los números naturales para dar sentido a la situación indicada en el problema, sin lograrlo (véase en la Figura 1 que ella no escribe 62 centímetros sino 62 metros). De acuerdo con las distinciones de Centeno (1988) en torno a los errores fundamentales detectados en la investigación previa, una clave de esta distorsión la constituye el ignorar la presencia del punto decimal, interpretando a partir de ello que el cero situado a la izquierda de dichas medidas es irrelevante. Presumiblemente, al desconocer Francisca el cero seguido del punto decimal en el resultado del algoritmo, suprimió el reconocimiento de una parte entera (expresada por el cero) y de una parte decimal en tal número, manteniendo, así, la designación final de metros.

Al triangular las tareas de naturaleza análoga, nos llamó la atención el contraste entre las Tareas 3 y 4 , ya que, en esta última, lo escrito por la joven fue adecuado $(0.76 \mathrm{~m})$, aunque 
esas medidas estaban conformadas, de modo similar, a las de la Tarea 3 (es decir, la parte entera de esos números estaba representada por el cero). Supuestamente, por tratarse de medidas ligadas al cuerpo humano y por eso, ser más familiares para ella (en la Tarea 4), Francisca efectuó una adecuada interpretación y resolución de la suma de decimales involucrada en el mencionado problema; esta situación la consideramos ligada al escrito de Freudenthal (1983), quien señala que el primer instrumento de medida que él ha identificado en los niños son los pasos, agregando a ello que hay una gran variedad de instrumentos de medida vinculados con el cuerpo humano tales como pulgadas, dedos, palmas, pies, entre muchos otros, los que son frecuentemente usados por los adultos, en distintas culturas.

El tercer error correspondió a la Tarea 7 de la Figura 1, en la que la joven escogió, (de acuerdo a las convenciones de México), la lectura adecuada del decimal: ocho kilómetros, ciento ochenta y cinco metros, conforme al sentido nacionalmente otorgado al punto decimal. Al mismo tiempo, canceló dicha respuesta escogida, al seleccionar también la expresión que corresponde a la lectura de un número natural: ocho mil ciento ochenta y cinco kilómetros (según las ya aludidas convenciones). Tal vez, Francisca se apoyó en una indebida interpretación de la expresión decimal dada como si fuese un entero positivo (véase la Figura 1). En ello, supuestamente consideró equivalentes la adecuada expresión decimal y la del número natural incorrecto de la Tarea 7; de ser así, nuevamente distorsionó la muchacha el sentido del punto decimal inserto en el número, al comienzo de este problema aritmético, aunque el cero no precedió aquí al punto decimal.

Con respecto a las demás tareas del cuestionario, la joven comparó satisfactoriamente dos distancias y estableció cuál de ellas era la mayor (Tarea 2), multiplicó y dividió correctamente los números decimales involucrados en los respectivos problemas (Tareas $5 \mathrm{y}$ 6). En la Tarea 8, Francisca escogió la cantidad de dinero adecuada y la expresó en pesos y centavos para hacerla corresponder a la lectura indicada; el uso de la moneda fue para ella muy frecuente debido a sus actividades laborales en la papelería, situación que no sólo la identifica como la persona que ejerce cierto trabajo en la sociedad de la que forma parte, sino que revela su condición de joven adulta en proceso de formación matemática básica. Al triangular las actividades del cuestionario, contrastando el acierto de la Tarea 8 con el error de la Tarea 7, interpretamos que el uso del dinero le facilitó optar por la expresión numérica correcta y fijar adecuadamente el punto decimal, circunstancia que permite corroborar cuán importante es la familiaridad que presente cierta tarea para que ésta resulte satisfactoriamente resuelta. 


\subsection{Resultados finales de la entrevista}

En la entrevista se reconstruyeron las Tareas 3, 4, 5 y 6 del cuestionario (a las que hemos designado como las Tareas A, B, C y D de la entrevista), con el propósito de ampliar la información que habíamos reunido en ellas originariamente, en torno a la adición, sustracción, multiplicación y división de números decimales, intentando constatar los supuestos derivadas del cuestionario. Además, se incluyeron otros cuatro problemas; en dos de estas nuevas tareas Francisca tuvo que comparar entre sí algunas medidas, en tanto que en las dos tareas restantes la entrevistada elaboró problemas, aplicando una suma y una resta de decimales, con el propósito de que ella pudiera evidenciar qué referentes concretos son de mayor relevancia, en su caso. Véase en la Figura 2 cada nuevo problema y su respectiva respuesta final.

\begin{tabular}{|c|c|}
\hline Tarea & Elaboración de Francisca \\
\hline $\mathrm{E}$ & 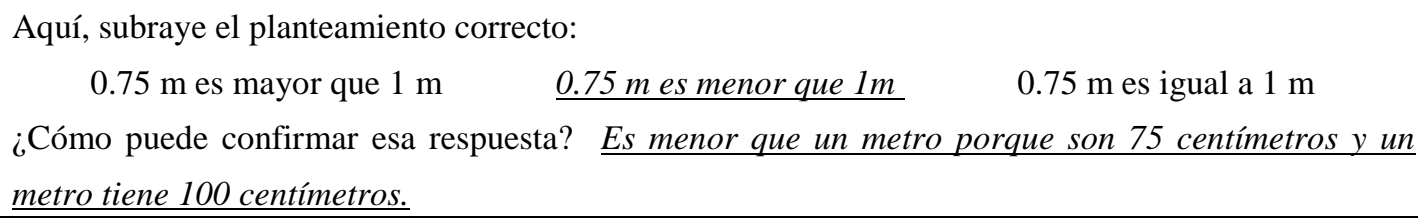 \\
\hline $\mathrm{F}$ & $\begin{array}{l}\text { Ahora, coloque una cruz junto a la expresión correcta: } \\
\quad 1.5 \text { litro es igual a } 1 \text { litro } \quad \text { - } 1.5 \text { litro es menor que } 1 \text { litro } \quad \times 1.5 \text { litro es mayor que } 1 \text { litro } \\
\text { ¿Por qué? Es más que un litro porque es medio litro más. }\end{array}$ \\
\hline G & $\begin{array}{l}\text { Escriba un problema aritmético que contenga una suma de decimales y resuélvalo. } \\
\text { Mi papá me dio } \$ 12.50 \text { y mi abuela me dio } \$ 8.50 \text {. ¿Cuánto dinero tengo? } \\
\underline{\text { Respuesta: } \$ 21.00}\end{array}$ \\
\hline $\mathrm{H}$ & 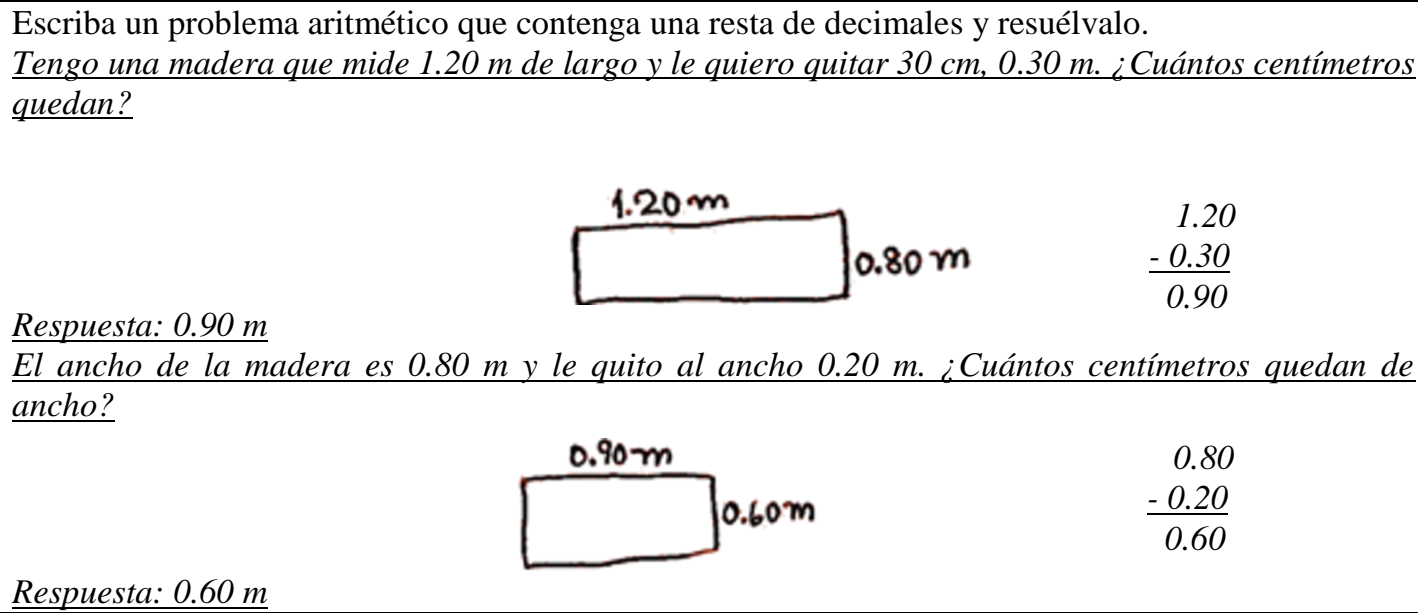 \\
\hline
\end{tabular}

Figura 2 - Las soluciones de Francisca ante las tareas exclusivas de la entrevista Fuente: contenidos producidos en la investigación de las autoras

\subsection{Análisis del diálogo desarrollado en la entrevista}

La dinámica global de la entrevista consistió en la presentación de cada uno de los ocho problemas incluidos en este instrumento, solicitándosele a Francisca que los leyera en 
voz alta para confirmar qué procesos de decodificación de los decimales desarrollaba. Luego, ella reflexionaba en silencio y escribía una operación cuando no la podía resolver mentalmente, comunicando, después los cálculos efectuados, para establecer el resultado de la tarea. El diálogo que promovía la entrevistadora consistía en explorar las dificultades cognitivas que eventualmente hubiese enfrentado la entrevistada, a la par que le requería a ésta justificar sus respuestas. Por último, la entrevistadora le pedía a la joven que escribiera lo que ya había expresado oralmente. A pesar de que el desenvolvimiento de Francisca en la entrevista fue globalmente atento y cuidadoso, incurrió en algunos errores de naturaleza diversa (de escritura y de lectura de decimales, de interpretación del punto decimal, entre los más importantes). La joven entrevistada dio muestras de que los problemas le resultaban casi siempre familiares y los resolvió comúnmente con soltura.

En las cuatro tareas del cuestionario reconstruidas en la primera sesión de trabajo de este instrumento, ella seleccionó de modo adecuado las operaciones idóneas para cada problema y, casi siempre, las resolvió correctamente. Asimismo, algunos de los errores cometidos por Francisca en el cuestionario reaparecieron en la lectura de decimales asociados a la medida de las patas de las mesas, expresadas por Francisca como cero setenta y cinco metros y la mención de producir una nueva mesa cuyas patas midan cero trece metros menos, lo cual fue expresado por la joven como cero trece metros menos [extensa pausa]... voy a restar trece metros (Tarea A de la entrevista, Tarea 3 del cuestionario). La entrevistadora introdujo, entonces, la convención nacional en torno a la lectura de esas expresiones decimales y, a partir de ello, Francisca re-significó esas notaciones y se esforzó por tomar en cuenta dicha convención (aunque no siempre lo logró, en el transcurso del diálogo de la entrevista).

En los dos problemas exclusivos de la entrevista, en los que Francisca debía comparar medidas (Tareas E y F), surgieron referencias a una medida entera en la justificación de la primera actividad (por conversión de 1 metro en 100 centímetros) y, en la segunda situación, una comparación con una fracción (allí donde Francisca reconoció implícitamente como equivalentes 0.5 litro y medio litro). Estos espontáneos procesos de significación que le permitieron a la entrevistada otorgar sentido a los decimales (en asociación con los números naturales o con las fracciones) están también ligados a otras respuestas de la entrevista.

En la Tarea D de la entrevista (Tarea 6 del cuestionario, donde tuvo una respuesta decimal adecuada) fueron notorias las dificultades de lectura de Francisca respecto a 1.5 litro de laca, pero mayor fue el conflicto cognitivo manifiesto en la aplicación del algoritmo de la división de decimales. El diálogo que sostuvo con la entrevistadora permitió a la joven, luego 
de prolongados silencios, dividir quince entre cinco [según ella expresó y realizó luego un cálculo mental] ... es 3 ... [la entrevistadora preguntó: ¿tres? ... la tercera parte... [la entrevistadora nuevamente preguntó: ¿tercera parte de qué?] ... de litro y medio, respondió Francisca, con esfuerzo, después de hacer la conversión de la parte decimal de 1.5 a expresión fraccionaria; así, Francisca re-significó adecuadamente el número dado en esta tarea.

En las dos actividades de la entrevista en las que le pedimos a la muchacha la elaboración de problemas verbales (Tareas G y H). La entrevistada seleccionó, en primer término, una situación de uso de dinero (Tarea G), en tanto que en el problema de medición que ella ideó se evidenciaron algunas dificultades asociadas tanto a la configuración del texto como a la designación y lectura de los correspondientes decimales.

A continuación, ante la imposibilidad de presentar todo el diálogo desarrollado en la entrevista, tan sólo reproducimos, con mayor detalle, la tarea en la que los intercambios verbales son más extensos y donde se destacan procesos importantes de elaboración o asignación de sentido a los números decimales, tanto como ciertas dificultades cognitivas y algunos errores evidenciados por la entrevistada. Para presentar los pasajes selectos de la entrevista, hemos tenido que respetar la integración natural del diálogo abajo transcripto.

\subsubsection{Análisis de la Tarea $\mathrm{H}$ - segunda parte de la entrevista}

Aquí, identificamos las elaboraciones cognitivas más esclarecedoras de los procesos de significación y de los referentes concretos primordiales para Francisca. En el inicio de esta actividad, la entrevistadora le preguntó a la entrevistada qué nuevas situaciones de aplicación de los números decimales se podrían proponer, aparte del uso del dinero que ella ya había expresado anteriormente (Tarea G) y que procuraba reiterar en la Tarea H. Ante tal interrogante, y con ostensibles dificultades, Francisca optó por la utilización de medidas de longitud expresadas mediante el sistema métrico decimal.

Francisca: Yo usaría metros y centímetros.

Entrevistadora: ¿Cómo los usarías?

Francisca: [Silencio prolongado.] Sería un metro y veinte centímetros ... [hizo una pausa muy extensa] y ... [nuevamente dudó y se detuvo] ... uno punto decimal ... [no concluyó] ...

Entrevistadora: ¿Cuál sería la situación que tú quieres plantear con un metro y veinte centímetros, haciendo una resta? ... ¿Cuál sería la situación que has vivido o visto? ... ¿Con tela? ¿Con madera? ... [Después de cada pregunta Francisca guardó silencio.] ... ¿Qué otra cosa pudiera ser?

Francisca: Con madera [se demoró mucho en responder, en tanto pensaba]. 
La entrevistada comenzó a escribir el texto del problema, ante lo cual la entrevistadora reanudó el diálogo, sugiriéndole que explicitase cómo había organizado la tarea y cuál era la pregunta que en la misma se formulaba.

Francisca: Tengo una madera que mide uno, veinte centímetros y le quiero quitar... [ella se mantuvo en un prolongado silencio].

Entrevistadora: Vuelve a leer el decimal, por favor. ¿Esa medida corresponde al largo?

Francisca: Mide un metro, veinte centímetros de largo y le quiero quitar... treinta centímetros... [la entrevistada se calló].

Entrevistadora: ¿A cuántos metros corresponde la última medida?

Francisca: Cero metros, treinta centímetros.

En el diálogo restante, Francisca produjo muchas pausas y mostró signos reiterados de duda. Después de resolver el problema cuya elaboración acabamos de presentar (véase la Figura 2), la entrevistadora le propuso tomar también en cuenta el ancho de la madera, circunstancia a partir de la cual la entrevistada escribió lentamente el segundo texto de la Tarea H, en cuya solución desarrolló otra resta de decimales e indicó la nueva respuesta.

Globalmente, en la Tarea H llama la atención que verbalizó y luego escribió $30 \mathrm{~cm}$ y $0.30 \mathrm{~m}$ como expresiones reconocidas tácitamente equivalentes, circunstancia que se enfatizó bastante durante la entrevista, ya fuese hablándolo o escribiéndolo, cuando Francisca necesitaba fijar correspondencias y procesos de significación entre decimales y naturales. Por otra parte, la última pregunta que formuló, en esa misma tarea, la expresó en centímetros (¿Cuántos centímetros quedan de ancho?), aunque pareció realizar mentalmente la correspondiente conversión de centímetros a metros, ya que continuó expresándose por escrito en metros, según lo evidencia la solución del problema. Todo ello denota que su actitud de duda ostensible, en la Tarea $\mathrm{H}$, estuvo más bien vinculada a que el referente concreto de medición de longitudes que la joven escogió le resultó menos familiar que el referente de uso de dinero (Tarea $\mathrm{G}$ ), en torno al cual ella exhibió una evolución bastante consistente, acorde con la propia naturaleza de sus actividades laborales.

\section{Conclusiones}

Una característica notable del desempeño de Francisca en la entrevista lo constituyeron sus conversiones de expresiones decimales en enteras positivas y viceversa; en menor medida, la joven apeló a la conversión de algunas expresiones decimales en fraccionarias, las que eran identificadas de una manera coloquial o informal; ambas producciones exhibieron el desarrollo de poderosos procesos de significación respecto a los números decimales, a través de los cuales la entrevistada se esforzó por dotar de contenidos 
semánticos a éstos, mediante sus posibles relaciones con los naturales y las fracciones. Es así como interpretamos el uso reiterado de expresiones equivalentes durante la entrevista, lo que constituyó una de las claves de la asignación de sentido, por la cual la joven decodificó las situaciones involucradas en los problemas y las correspondientes expresiones numéricas.

Los procesos de pensamiento más relevantes surgidos de la entrevista fueron los que respaldaron la adecuada elección de la operación correspondiente a cada problema, por parte de la joven, quien los hacía manifiestos de un modo muy directo. A veces, esos procesos de pensamiento le permitieron anticipar el efecto que generaría la operación en el resultado de la misma (en la Tarea A, por ejemplo, justificó que la nueva mesa se haría más chica, al restar).

En cuanto a los referentes concretos privilegiados por esta joven, al elaborar tareas propias con respecto a los números decimales, en primera instancia se destacó la situación de uso de dinero y, en segundo término, la utilización de algunas medidas de longitud representadas a través del sistema métrico decimal.

Las dificultades cognitivas detectadas en Francisca fueron la interpretación posicional y la notación adecuada del punto decimal, especialmente, al restar y dividir decimales. En íntima conexión con tales dificultades, pudimos identificar algunos errores (de lectura, de escritura y operacionales), los que guardan relación con nociones posicionales ambiguas o inestables de la joven (estos datos son coincidentes con los planteados por CENTENO, 1988).

En general, mediante la entrevista dimos respuesta a los supuestos asociados a las Tareas 3 y 7 del cuestionario exploratorio, al identificar algunas dificultades cognitivas de Francisca relacionadas con la lectura e interpretación de ciertas notaciones decimales. Con respecto al supuesto ligado a la Tarea 4 del cuestionario, el mismo deberá ser confirmado en indagaciones futuras, con otros sujetos.

Asimismo, en la entrevista Francisca se enfrentó con situaciones familiares (conforme a BELL; SWAN; TAYLOR, 1981), desde donde ella pudo fortalecerse y ofrecer estrategias adecuadas para la solución de los problemas. El diálogo con la entrevistadora proveyó un espacio social de intercambio en el que pudo replantear sus propias elaboraciones, cuando éstas eran ambiguas o erróneas. En un futuro, sería conveniente que algunas de esas actividades pudieran desarrollarse en el aula, entre los adultos que participan en el mismo grupo escolar, para favorecer la superación de los errores sistemáticos en los que suele incurrir el sujeto del aprendizaje (según STEINLE y STACEY, 2004) y comprobar el alcance de una enseñanza orientada a fortalecer el pensamiento (conforme a OKAZAKI, 2003). 


\section{Referencias}

BELL, A.; SWAN, M.; TAYLOR, G. Choice of operation in verbal problems with decimal numbers. Educational Studies in Mathematics, Dordrecht, The Netherlands, v. 12, p. 399-419, 1981.

BOUFI, A.; SHAFTOURU, F. Supporting students' reasoning with decimal numbers: A study of a classroom's mathematical development. In: CONFERENCE OF THE INTERNATIONAL GROUP FOR THE PSYCHOLOGY OF MATHEMATICS EDUCATION, 26., 2002, Norwich. Proceedings... Norwich, England: COCKBURN, A.; NARDI, E., 21 al 26 de julio de 2002. p. 153-160.

BROITMAN, C.; ITZCOVICH, H.; QUARANTA, M. E. La enseñanza de los números decimales: El análisis del valor posicional y una aproximación a la densidad. Revista Latinoamericana de Investigación en Matemática Educativa, Ciudad de México, México, v. 6, n. 1, p. 5-26, 2003.

BRUNER, J.; HASTE, H. La elaboración de sentido. 1. ed. En español. Barcelona: Paidós, 1990. $192 \mathrm{p}$.

CARPENTER, T.; FENNEMA, E.; ROMBERG, T. Toward an unified discipline of scientific inquiry. In: CARPENTER, T.; FENNEMA, E.; ROMBERG, T. (Ed.). Rational numbers. An integration of research. Hillsdale, USA: Ed. Lawrence Erlbaum Associates Publishers, 1993. p. 2-11.

CENTENO, J. Números decimales ¿Por qué? ¿Para qué? 1. ed. Madrid: Síntesis, 1988, 212 p.

COHEN, L.; MANION, L. Métodos de investigación educativa. 1a. ed. En español. Madrid: La Muralla, 1990. $502 \mathrm{p}$.

DELIYANNI, E.; ELIA, I.; PANAOURA, A.; GAGATSIS, A. A structural model for the understanding of decimal numbers in primary and secondary education. In: CONFERENCE OF THE INTERNATIONAL GROUP FOR THE PSYCHOLOGY OF MATHEMATICS EDUCATION, 33., 2009, Thessaloniki. Proceedings... Thessaloniki, Greece. TZEKAKI, M.; KALDRIMIDOU, M.; SAKONIDIS, H., 19 al 24 de julio de 2009, p. 393-400.

DUCROT, O.; TODOROV, T. Diccionario enciclopédico de las ciencias del lenguaje. 7. ed. En español. México: Siglo XXI Editores, 1981, 421 p.

FREUDENTHAL,H. Didactical Phenomenology of Mathematical Structures. 1. ed. Dordrecht/London/Moscow: Kluwer Academic Publishers, 1983. 586 p.

GARCÍA CARRASCO, J. Educación de adultos. 1ª ed. Barcelona: Editorial Ariel, 1997. 340 p.

GIMENO, J.; PÉREZ, A. Comprender y transformar la enseñanza. 9a .ed. Madrid: Ediciones Morata, 2000. $448 \mathrm{p}$.

HUNTER, R.; ANTHONY, G. "Sensing": Supporting student understanding of decimal knowledge. In: ANNUAL CONFERENCE OF THE INTERNATIONAL GROUP FOR THE PSYCHOLOGY OF MATHEMATICS EDUCATION, 27., 2003, Hawaii. Proceedings... Hawaii: PATEMAN, N. A.; DOUGHERTY, B. J.; ZILLIOX, J. T., 13, al 18 de julio de 2003. p. 41-48.

JÓIA, O. Cuatro preguntas sobre la educación matemática de jóvenes y adultos. In: Conocimiento matemático en la educación de jóvenes y adultos. Santiago: Ed. UNESCO - SANTIAGO, 1997. p. 27-34.

KIEREN, T. Rational and fractional numbers: From quotients fields to recursive understanding. In: CARPENTER, T.; FENNEMA, E.; ROMBERG, T. (Ed.). Rational numbers. An integration of research. Hillsdale: Lawrence Erlbaum Associates, Publishers, 1993. p. 49-84. 
MESSINA, G. La educación básica de adultos: La otra educación. $1^{\text {a }}$. ed. Santiago, Chile: UNESCO-OREALC, 1993. 208 p.

NESHER, P. Levels of description in the analysis of addition and subtraction word problems. In: CARPENTER, T.; MOSER, J.; ROMBERG, T. (Ed.). Addition and subtraction: A cognitive perspective. Hillsdale: Ed. Lawrence Erlbaum Associates Publishers, 1982. p. 25-38.

NESHER, P. Solving multiplication word problems. In: LEINHARDT, G; PUTNAM, R.; HATTRUP, R. (Ed.). Analysis of arithmetic for mathematics teaching. Hillsdale: Ed. Lawrence Erlbaum Associates Publishers, 1992. p. 189-219.

OKAZAKI, M. Characteristics of 5th graders' logical development through learning division with decimals. In: CONFERENCE OF THE INTERNATIONAL GROUP FOR THE PSYCHOLOGY OF MATHEMATICS EDUCATION, 27., 2003, Hawaii. Proceedings... Hawaii: PATEMAN, N. A.; DOUGHERTY, B. J.; ZILLIOX, J. T., 13 al 18 de julio de 2003. p. 413-419.

SCHWARTZ, H.; JACOBS, J. Sociología Cualitativa. Método para la reconstrucción de la realidad. $1^{a}$. Ed. En español. Ciudad de México: Trillas, 1984, 558 p.

STEINLE, V.; STACEY, K. Grade-related trends in the prevalence and persistence of decimal misconceptions. In: CONFERENCE OF THE INTERNATIONAL GROUP FOR THE PSYCHOLOGY OF MATHEMATICS EDUCATION, 27., 2003, Hawaii. Proceedings... Hawaii: PATEMAN, N. A.; DOUGHERTY, B. J.; ZILLIOX, J. T., 13 al 18 de julio de 2003. p. 259-266.

STEINLE, V.; STACEY, K. Persistence of decimal misconceptions and readiness to move expertise. In: CONFERENCE OF THE INTERNATIONAL GROUP FOR THE PSYCHOLOGY OF MATHEMATICS EDUCATION, 28., 2004, Bergen. Proceedings... Bergen: Johnsen, M.; Berit, A., 14 al 18 de julio de 2004. p. 225-232.

TAYLOR, S.; BOGDAN, R. Introducción a los métodos cualitativos de investigación. La búsqueda de significados. $1^{a}$. ed. En español. Buenos Aires: Paidós, 1987. 344 p.

VALDEMOROS, M. La constancia de la unidad en la suma de fracciones: Estudio de caso. In: HITT, F. (Ed.). Investigaciones en Matemática Educativa II. México: Ed. Editorial Iberoamérica, 1998. p. 465-481.

VALDEMOROS, M.; RUIZ, E. F. El caso de Lucina para el estudio de las fracciones en la escuela primaria. Revista Latinoamericana de Investigación en Matemática Educativa, Ciudad de México, México, v. 11, n. 1, p. 127-157, 2008.

VERGNAUD, G. A classification of cognitive tasks and operations of thought involved in addition and subtraction problems. In: CARPENTER, T.; MOSER, J.; ROMBERG, T. (Ed.). Addition and subtraction: A cognitive perspective. Hillsdale: Lawrence Erlbaum Associates Publishers, 1982. p. 39-59.

WOODS, P. Investigar el arte de la enseñanza. El uso de la etnografía en la educación. 1. ed. En español. Barcelona: Editorial Paidós, 1987. 232 p.

Submetido em Abril de 2016. Aprovado em Novembro de 2016. 


\section{ERRATA}

Na página 699, Onde se lia:

"Marta Elena Valdemoros Álvarez

Elena Fabiola Ruiz Ledesma"

\section{Leia-se}

"Marta Elena Valdemoros-Álvarez Elena Fabiola Ruiz-Ledesma"

Bolema (2018) 32(60): i-i 